\title{
Review of: "Nutritional evaluation and transcriptome analyses of short-time germinated seeds in soybean (Glycine max L. Merri.)"
}

Xiujun Lu

Potential competing interests: The author(s) declared that no potential competing interests exist.

In this study, the authors investigated the nutritional value and genome-wide gene expression patterns of whole seeds germinated for short-time by HPLC, RNA-seq and related techniques. This paper provides referable data on nutritional and gene regulation mechanisms for transcriptional regulation of germination in soybean seeds, which has some scientific and informative implications.

There are several problems in this study:

1 Because both "short time germinated seeds" and conventional "germinated seeds" can cause changes in storage proteins, carbohydrates, photosynthesis and isoflavone pathways, it is necessary to clarify the difference between the two. The title of the article uses the term "short time germinated seeds", but the difference between "short time germinated" and "germinated" in general, as well as the characteristics and significance of the choice of "short time germinated" in this article, are not sufficiently introduced in the introduction.

2.1 The article mentions "No signifcant change was observed for total isofavone and glucosides-conjugated isofavones in diferent cultivarss (Fig.1D)" in the paragraph "Major nutrients evaluation of germinated soybean seeds". Is there any experimental data to prove that there is no difference in the isoflavone index of all 3 varieties? If available, it is recommended to supplement to the figures and be labeled in the main text.

2.2 “Fig.1D, isofavone (daidzin, glycitin, genistin, daidzein, glycitein, and genistein) content in germinated seeds of three independent cultivars (ZD41, JHD, and J58) are compared between control (0 h) and germinated for $36 \mathrm{~h}$. "Is each isoflavone index the average of the three varieties measured separately? Or do you choose to replace one of the indicators with another because there is no significant difference between the indicators of the 3 species? There is some ambiguity here, please elaborate on the reasons for not expressing them separately.

3 Both seed morphology (Fig.1A) and RNA-Seq were determined for 3 samples (0 HAG, 18 HAG, and 36 HAG) in the article, but PC, WSPC, and isofavone were determined for 2 samples ( $0 \mathrm{~h}$ and $36 \mathrm{~h}$ ) (Fig.1B-D). For the deletion and retention of "18 HAG", sufficient experimental data and analytical descriptions should be given.

4 There are two ways to express the sample time points in the article, $0 \mathrm{~h}, 18 \mathrm{~h}$, and $36 \mathrm{~h}$ and 0 HAG, 18 HAG, and 36 HAG, both expressions will be ambiguous to the readers, it is suggested to keep one of them 
uniformly throughout the article including the pictures.

5 With the continuous updating of the soybean genome, the annotation of the soybean transcriptome will vary. The version number of the reference soybean genome and the number of annotated genes are not indicated in the RNA-Seq results in the article, and this information is the basis for the subsequent analysis of the number of differential genes and the annotation results, so it is recommended to add a description. 6 Data analysis was shallow. The analysis of soybean nutrient indicators and transcriptome data in the article is mainly a simple description of the information easily available in the pictures, but less analysis of the scientific significance of the indicators or the patterns of expression changes. For example, the paragraph "GO analysis of DEGs" is too detailed in listing the biological processes after photosynthesis mobilization, which can be obtained directly from Figure 3A, resulting in a lengthy paragraph. It is thought that the analysis of the results should be increased and the description of the pictures should be reduced, which can improve the efficiency of the reader in obtaining effective information.

7 During seed germination, dramatic changes in certain metabolic pathways can be expected, such as lipid degradation, carbohydrate synthesis, and hormone metabolism. A large number of differentially expressed genes involved in these biological processes were detected in this study, but they were not elaborately analyzed. For example, a particular gene can positively or negatively regulate a metabolic pathway, thus, a general description of a gene's up- or down- expression is meaningless. I suggest that the authors focus on the expression of some key genes in the pathway.

8. The discussion lacks appropriate references. For example, in the last paragraph of the discussion, is the conclusion about the role of the "phenylpropanoid pathway" in seed germination obtained from this paper or from other studies? If it is a conclusion obtained from this paper, are there any other studies with similar or contradictory results? If it is a reference to the results of other studies, this section should be labeled. 